\title{
Review
}

\section{Oligosaccharides in infant formula: more evidence to validate the role of prebiotics}

\author{
Yvan Vandenplas $^{1 *}$, Irina Zakharova ${ }^{2}$ and Yulia Dmitrieva ${ }^{2}$ \\ ${ }^{1}$ Pediatric Gastroenterology, UZ Brussel, Vrije Universiteit Brussel, Laarbeeklaan 101, 1090 Brussels, Belgium \\ ${ }^{2}$ Pediatric Department, Russian Medical Academy of Postgraduate Education, Moscow, Russia
}

(Submitted 7 November 2014 - Final revision received 29 January 2015 - Accepted 17 February 2015 - First published online 2 April 2015)

\begin{abstract}
The gastrointestinal (GI) microbiota differs between breast-fed and classic infant formula-fed infants. Breast milk is rich in prebiotic oligosaccharides (OS) and may also contain some probiotics, but scientific societies do not recommend the addition of prebiotic OS or probiotics to standard infant formula. Nevertheless, many infant formula companies often add one or the other or both. Different types of prebiotic OS are used in infant formula, including galacto-oligosaccharide, fructo-oligosaccharide, polydextrose and mixtures of these OS, but none adds human milk OS. There is evidence that the addition of prebiotics to infant formula brings the GI microbiota of formula-fed infants closer to that of breast-fed infants. Prebiotics change gut metabolic activity (by decreasing stool pH and increasing SCFA), have a bifidogenic effect and bring stool consistency and defecation frequency closer to those of breast-fed infants. Although there is only limited evidence that these changes in GI microbiota induce a significant clinical benefit for the immune system, interesting positive trends have been observed in some markers. Additionally, adverse effects are extremely seldom. Prebiotics are added to infant formula because breast milk contains human milk OS. Because most studies suggest a trend of beneficial effects and because these ingredients are very safe, prebiotics bring infant formula one step closer to the golden standard of breast milk.
\end{abstract}

Key words: Breast-feeding: Infant feeding: Galacto-oligosaccharides: Infant formula: Oligosaccharides: Prebiotics

Prebiotic oligosaccharides (OS) are the third most prevalent component in breast milk, but they are virtually absent in cow's milk ${ }^{(1)}$. Human milk OS are comprised of a complex mixture of glycan compounds. Prebiotics are non-digestible food ingredients that stimulate the growth and/or activity of bacteria in the digestive system in ways that are claimed to be beneficial to human health. It is well known that the gastrointestinal microbiota develops in different ways in breast-fed and standard formula-fed infants ${ }^{(2)}$. There are many aspects about the composition of breast milk and that of infant formula that contribute to these differences, including their carbohydrate, protein, Fe and phosphor content. The gastrointestinal microbiota is considered to be an organ within an organ that contributes to host nutrition, the developmental regulation of intestinal angiogenesis, protection from pathogens and the development of immune response ${ }^{(1,3)}$.

The concept of dietary modulation of the human colonic microbiota with prebiotics was developed about 20 years ago $^{(4)}$. In infant formula, the main prebiotics that are used include galacto-oligosaccharide (GOS), fructo-oligosaccharide (FOS) and/or polydextrose (PDX). There is strong clinical support that GOS, which have some similarities to human milk OS, are beneficial for both digestive and immune health ${ }^{(5)}$. This was also shown for inulin-type fructans ${ }^{(1)}$. The prebiotic effect is defined as the selective stimulation of growth and/ or activity(ies) of one or a limited number of microbial genus(era)/species in the gut microbiota that confer(s) health benefits to the host ${ }^{(5)}$.

\section{Prebiotics and bifidogenic effect, gut metabolic activity} and stool consistency

Prebiotics are added to infant formula with the aim of achieving a bifidogenic effect on the gastrointestinal microbiota of the host. This effect is by definition global. Protein, in terms of both source (such as whey or $\alpha$-lactalbumin) and

Abbreviations: FOS, fructo-oligosaccharide; GOS, galacto-oligosaccharide; lcFOS, long-chain fructo-oligosaccharide; OS, oligosaccharide; PDX, polydextrose; RCT, randomised controlled trial; sIgA, secretory IgA; scGOS, short-chain galacto-oligosaccharide.

*Corresponding author: Professor Y. Vandenplas, fax: + 32247757 83, email yvanvandenplas@uzbrussel.be 
amount, lactose, low phosphor and $\mathrm{Fe}$, lipids ( $\beta$-palmitate) and nucleotides are all known to have a bifidogenic effect ${ }^{(6)}$.

\section{Prebiotics and the bacterial composition of the gastrointestinal microbiota}

The type of prebiotic supplement may have a different bifidogenic effect, seeing as short-chain prebiotics are mainly fermented in the caecum and ascending colon. Long-chain OS are fermented in the entire colon ${ }^{(7)}$. The transcriptional effect of a semi-synthetic medium containing GOS, which, like human milk, contains a large amount of lactose and galactose, on the Bifidodobacterium longum transcriptome has revealed substantial similarity to that of human milk ${ }^{(8)}$ GOS and lactulose support the most favourable growth characteristics, whereas relatively poor Lactobacillus and Bifidodobacterium growth was observed with inulin, maltodextrin and $\mathrm{PDX}^{(9)}$. When comparing different formulas, including oligofructose/FOS (50:50, 4 or 8g/1), GOS/FOS (90:10, $8 \mathrm{~g} / \mathrm{l})$ and standard formula, the bacterial composition in the first two formulas was closer to that of the breast-fed pattern ${ }^{(10)}$. The addition of a GOS/FOS mixture or of GOS only to infant formula has a stimulating effect on the growth of Bifidodobacterium ${ }^{(11-18)}$. GOS alone was shown to increase Lactobacillus ${ }^{(14,15)}$. The proportion of Bifidodobacterium was higher in the prebiotic group, but not significantly ( $P=0 \cdot 262)$, whereas faecal clostridia was significantly lower $(P=0 \cdot 042)^{(12)}$. Similar results were reported with a GOS/FOS mixture: there was a significant increase in Bifidodobacterium and a decrease in clostridia ${ }^{(15)}$. Bifidodobacterium breve growth was higher and Clostridium difficile was lower in 365 infants that were fed GOS at $4 \mathrm{~g} / \mathrm{l}$ in infant formula and $5 \mathrm{~g} / \mathrm{l}$ in follow-up formula as compared to control formula(13). Supplementation with other prebiotics, such as PDX and GOS, led to comparable results ${ }^{(19)}$

In preterm infants, FOS-supplemented formula has been shown to have positive effects on gut microbiota development: the number of Bifidodobacterium and proportion of infants colonised were significantly higher in the study group ( $P=0.032$ and $P=0.03$, respectively), which resulted in a significant reduction of Escherichia coli and Enterococci $(P=0.029 \text { and } P=0 \cdot 025 \text {, respectively })^{(20)}$.

The magnitude of the prebiotic impact may be age dependent, seeing as the bifidogenic effect has been reportedly larger when administration was begun in early infancy ${ }^{(21)}$. However, GOS supplementation also significantly increased Bifidodobacterium numbers in vivo and in vitro in women and men who were more than 50 years old ${ }^{(22)}$. Prebiotics have also been shown to have the potential to reduce disturbances in the gut microbiota that were induced by antibiotics $^{(23)}$. Changes in the relative abundance of bacteria after antibiotic treatment and the growth of Bifidodobacterium and Lactobacillus after the addition of GOS were dependant on the type of antibiotic and dose amount ${ }^{(23)}$.

No local or systemic side effects were recorded ${ }^{(17)}$.

It can be concluded that the majority of prebiotics are able to influence the intestinal microbiota of young infants.

\section{Prebiotics and faecal $\mathrm{pH}$}

Faecal $\mathrm{pH}$ was lower $(P=0.01)$ in breast-fed and prebiotic formula-fed infants than it was in standard infant formula-fed infants ${ }^{(12)}$. Infant formula containing $0.24 \mathrm{~g} / 100 \mathrm{ml}^{(14)}$ and $0 \cdot 44 \mathrm{~g} / 100 \mathrm{ml}$ of $\mathrm{GOS}^{(13)}$ has been shown to bring faecal $\mathrm{pH}$ to a similar level as that of infants fed human milk. Intestinal $\mathrm{pH}$ modulates the intestinal environment by inhibiting or favouring the growth of different bacterial populations; a low intestinal $\mathrm{pH}$ results in a decrease of pathogenic microorganisms $^{(18)}$.

\section{Prebiotics and metabolic activity}

Feeding infants a GOS/FOS or a GOS-only formula has resulted in a similar effect on the metabolic activity of the microbiota as that found in breast-fed infants ${ }^{(11,13,24)}$. The changes in SCFA and lactate (and pH) in one study's prebiotic group represented a fermentation profile that was closer to that observed in breast-fed infants as compared to infants fed control formula ${ }^{(11)}$. Because SCFA represent the metabolic activity of intestinal microbiota ${ }^{(24)}$, these findings suggest that the gut microbiota of infants fed a prebiotic-supplemented formula develops similar to that in breast-fed infants.

Acetic acid, whose formation is consistent with Bifidodobacterium metabolism, was the major SCFA synthesised ${ }^{(25)}$. Purified GOS can be considered bifidogenic ${ }^{(25)}$. The effect of GOS on intestinal metabolic activity has been observed in a recent study: GOS supplementation resulted in a significant increase in total SCFA, higher acetic acid and lower butyric and propionic acids as compared to breast-fed infants ${ }^{(13)}$.

\section{Prebiotics and stool patterns}

Different levels of GOS (0.24 g/100 $\mathrm{ml}$ and $0.4 \mathrm{~g} / 100 \mathrm{ml})$ in infant formula increase stool frequency, as does breast milk ${ }^{(14-16)}$. Prebiotics increase the bacterial mass and the osmotic waterbinding capacity in the gut lumen. These actions increase stool weight and frequency, and they also soften the stool, which indirectly contributes to decreased transit time.

Compared to infants who received standard formula, infants who received prebiotic supplementation experienced softer stools that were similar to the consistency reported in breastfed infants ${ }^{(26)}$. Standard infant formula with $4 \mathrm{~g} / \mathrm{l}$ GOS had beneficial effects on both stool consistency and additional measures of gastrointestinal tolerance by newborn infants during the first 4 months of life $\mathrm{e}^{(27)}$. Infants fed a prebiotic GOS/FOS mixture had softer stools than those fed standard infant formula $(P=0.026)^{(12)}$. The infants that consumed PDX/GOS (1:1, $4 \mathrm{~g} / \mathrm{l})$ for $60 \mathrm{~d}$ had softer stools than the control group at all times $(P<0 \cdot 001)^{(19)}$. No differences in formula intake or infant fussiness or gassiness were observed. During study weeks 1 and 2 and at $60 \mathrm{~d}$ of age, stool consistency ratings were higher (i.e. softer stools) for infants in the PDX/GOS and GOS groups $v$. the control group and remained higher at $120 \mathrm{~d}$ for the PDX/GOS group (all $P<0 \cdot 05)^{(19)}$. In a systematic review, prebiotics were reported to have increased stool frequency but had no impact on stool consistency, the incidence 
of colic, spitting up/regurgitation, crying, restlessness or vomiting ${ }^{(28)}$. When comparing a mixture of oligofructose/ FOS $(50: 50 ; 4$ or $8 \mathrm{~g} / 1)$, GOS/FOS $(90: 10,8 \mathrm{~g} / \mathrm{l})$ and standard formula, the stool consistency of infants who were fed $8 \mathrm{~g} / 1$ of GOS/FOS-supplemented formula was closer to the breastfed pattern ${ }^{(10)}$

Follow-up formulas with only $5 \mathrm{~g} / \mathrm{l}$ GOS that were provided to infants aged 6 months to 1 year resulted in a higher frequency and softer consistency of stools ${ }^{(13)}$. Supplementation with $5 \mathrm{~g} / \mathrm{l}$ GOS did not alter stool frequency, but it did decrease stool consistency ${ }^{(17)}$.

Several prebiotic mixtures have been evaluated in different studies. In a systematic review about the effect of prebiotics on stool frequency and consistency ${ }^{(29)}$, four randomised controlled trials (RCT) demonstrated that the stool frequency of the prebiotic group was similar to that of breast-fed infants and was significantly higher than that of infants fed standard formula. Similar data were obtained regarding stool consistency: six RCT showed significantly softer stools in the prebiotic group $^{(29)}$. In a large study in 1130 infants, the stool consistency in the prebiotic group was softer than in the control group at 8,16 and 24 weeks $(P<0 \cdot 001)$ and closer to that of the breast-fed group ${ }^{(30)}$.

\section{Prebiotics and general health}

GOS has been shown to reduce $\mathrm{Ca}$ excretion in faeces in normal and gastrectomised rats, and thus to prevent osteope$\mathrm{nia}^{(31)}$. The addition of a specific mixture of $0.6 \mathrm{~g} / 100 \mathrm{ml}$ of short-chain galacto-oligosaccharide (scGOS) and long-chain fructo-oligosaccharides (lcFOS) in a 9:1 ratio to infant formula did not decrease cholesterol concentrations, although the levels in formula-fed infants were significantly lower than those in breast-fed infants ${ }^{(32)}$. One open trial of 342 healthy infants compared standard infant formula with a GOS/FOSenriched formula. The formulas were given for 6 months, and at the age of 12 months, the incidence of gastroenteritis was lower $(0 \cdot 12 \pm 0.04 v \cdot 0 \cdot 29 \pm 0.05$ episodes $/$ child per 12 months; $P=0.015)$, the number of children with more than three episodes tended to be lower (17/60 v. 29/65; $P=0.06)$ and the number of children with multiple antibiotic courses per year was lower $(24 / 60 \quad v .43 / 65 ; P=0.004)$ in the enriched-formula group ${ }^{(33)}$. Infants that were fed an scGOS/ lcFOS mixture were reported in another study to have fewer episodes of physician-diagnosed overall and upper respiratory tract infections $(P<0.01)$, fewer fever episodes $(P<0.00001)$ and fewer antibiotic prescriptions $(P<0.05)$ during the period of intervention and up to the age of 2 years ${ }^{(34,35)}$. According to these results, early dietary intervention with an OS prebiotic mixture had a protective effect against infections ${ }^{(34)}$. However, in other randomised clinical trials, these results have not been confirmed. In one large, randomised, double-blind, placebo-controlled trial performed in 830 healthy term infants in seven centres in five Western European countries, adding specific prebiotics to standard formulafeeding had no effect on reducing the number of fever episodes $^{(36)}$. These results are in alignment with those from another recent randomised, double-blind, placebo-controlled clinical trial that used only GOS as a prebiotic source in infant formula; in that study, no differences were observed in allergic manifestations up to 12 months of age, and there were no differences in the numbers of diarrhoea episodes or prescriptions of antibiotics ${ }^{(13)}$. This difference in results may be explained by differences in the study populations: in the last study, all of the infants were healthy term infants who lived in modern environments with a low risk of infectious disease, but this was not the case in some of studies described earlier. Further studies in populations with a higher incidence of infections, perhaps in developing countries, and further studies on the effects of prebiotics on allergy manifestations in infants with a high risk of atopy are needed ${ }^{(13)}$.

\section{Prebiotics and growth}

A transient increase in body weight was observed in children on prebiotics as compared to the control group during the first 6 months of follow-up ${ }^{(33)}$. Prebiotics in formula increased weight gain but had no impact on length or head circumference gain ${ }^{(12,28)}$. However, the quality of this evidence is compromised by imprecision, an inconsistency of results, the use of different study preparations and publication bias ${ }^{(28)}$. As a consequence, evidence for the benefit of symbiotics added to infant formula is equally limited.

A multi-centre, randomised, double-blind, placebo-controlled trial including 1130 healthy term infants showed similar mean weight, length, head circumference, skinfold thickness and arm circumference gains in all infants ${ }^{(35)}$. The skinfold thicknesses assessed in the breast-fed group at 8 weeks were strikingly larger than those in the formula-fed infants, whereas at 52 weeks, they were strikingly smaller ${ }^{(35)}$

Other studies have not observed these differences in growth parameters. One study that compared prebiotic-enriched to standard infant formula showed no group differences in growth rates from 14 to $120 \mathrm{~d}$ of age ${ }^{(36)}$. Discontinuation rates were not significantly different among the study groups. The tolerance of a formula enriched with FOS/GOS was excellent, and no differences in growth parameters were observed after 6 and 12 weeks $^{(12,34,36)}$. According to a systematic review from 2009, ten publications evaluated the effect of prebiotics on growth during the 1 st year of life ${ }^{(37)}$. None of these trials showed a significant difference in growth parameters; infants fed the supplemented formula had only slightly better weight gain (weighted mean difference $1.07 \mathrm{~g}, 95 \%$ CI $0.14,1.99$; four RCT) ${ }^{(37)}$. This is in agreement with the results obtained recently by Sierra et $a l .{ }^{(13)}$, which observed no significant differences in growth.

\section{Prebiotics and immune parameters}

A greater number of Bifidodobacterium may promote postnatal maturation of the immune system and thus have a protective effect against infections and allergies. The correlation between Bifidodobacterium and the amount of intestinal secretory IgA (sIgA) has been known for many years ${ }^{(12,38,39)}$. Prebiotic inulin and oligofructose clearly modulate immunological processes at the level of the gut-associated lymphoid tissue, and 
they may be associated with significant health benefits in infants and patients who have intestinal inflammatory diseases ${ }^{(40)}$. The prebiotic effect that was observed after the intervention period suggests that an immune modulating effect through modification of the intestinal microbiota may be the principal mechanism of action ${ }^{(36)}$. One area of research on modern infant formula focuses on the development of the immune system. The addition of long-chain PUFA is outside the topic of the present review, but they are shown to positively influence the development of the immune system ${ }^{(41)}$.

In practice, these different trial parameters are often combined, which makes it more difficult to know 'which parameter did what'. This has been observed in the RCT where $0.44 \mathrm{~g} / 100 \mathrm{ml}$ and $0.5 \mathrm{~g} / 100 \mathrm{ml}$ of GOS were added to infant and follow-up formulas, respectively; long-chain PUFA and nucleotides were also included in the standard formula ${ }^{(13)}$. The addition of a specific mixture of $0.6 \mathrm{~g} / 100 \mathrm{ml}$ of scGOS and lcFOS in a 9:1 ratio to infant formula showed that immune parameters at weeks 8 and 26 did not differ between the two groups ${ }^{(42)}$. The mixture of prebiotic os did not change the basal level of the measured parameters of the developing immune system in healthy infants with a balanced immune system during the first 6 months of life as compared to that in standard formula-fed infants and that in exclusively breast-fed infants ${ }^{(42)}$. No significant differences were detected between PDX/GOS supplementation and the control group in changes from baseline to 30 or $60 \mathrm{~d}$ for $\operatorname{sigA}{ }^{(19)}$. After 26 weeks of intervention with the same mixture, the concentration of sIgA was higher $(P<0.001)$ in the scGOS/lcFOS group $(719 \mu \mathrm{g} / \mathrm{g})$ than it was in the control group $(263 \mu \mathrm{g} / \mathrm{g})$, whereas the concentration in the breast-fed group was similar to that in the intervention group ${ }^{(15)}$. In another study, GOS reduced colitis by modulating the function and trafficking of natural killer cells in mice ${ }^{(43)}$.

There is only one study which provided evidence that GOS/ FOS supplementation induced a beneficial antibody profile ${ }^{(44)}$. A GOS/FOS mixture reduced the total Ig response and modulated the immune response to cow milk protein but left the response to vaccination intact ${ }^{(44)}$. In a recent multi-centre, randomised, double-blind, placebo-controlled trial carried out in 365 infants, sIgA concentration decreased in both groups, and there was a trend towards a lower decrease in the GOS group $^{(13)}$. This suggests a protective effect on the development of a gut mucosa immune response, given that the sIgA decrease was more stable over time in the infants who consumed the GOS-containing formula; it also reflects a similar behaviour to that observed in breast-fed infants ${ }^{(19,45)}$

\section{Prebiotics and allergies and atopic dermatitis}

Prebiotics were shown to reduce Ig free light chain concentrations in infants who were at risk for allergies ${ }^{(46)}$. GOS has been shown to block atopic dermatitis, such as skin lesions, in mice by at least partly inducing the production of IL-10 and suppressing the production of cytokines, such as IL-17, which are involved in skin inflammation ${ }^{(47)}$. In a large study in 259 high-risk infants, prebiotic supplements to formula-feeding reduced the development of atopic dermatitis at the age of
6 months ${ }^{(48)}$. However, systematic reviews have failed to prove the efficiency of prebiotics in atopic dermatitis prevention ${ }^{(49)}$. Early dietary intervention with OS has been shown to have a protective effect against allergy manifestations. When they were started early in life, OS (scGOS/lcFOS) had a protective effect against allergy manifestations in high-risk infants that lasted beyond infancy until the age of 5 years for atopic dermatitis and allergic rhinoconjunctivitis ${ }^{(49,50)}$. Although the intervention group had a $75 \%$ reduction in the prevalence of persistent wheezing ( $4.8 v .14 \%)$, no significance was shown $^{(49,50)}$. The specific scGOS/lcFOS mixture did, however, reduce the allergic reaction to ovalbumin in mice ${ }^{(50)}$. The use of GOS/FOS in dietary products has also been shown to possibly stimulate the adaptive immune response in a T helper 1 direction and subsequently inhibit infections and $\mathrm{T}$ helper 2-related immune disorders, such as allergies, in human subjects ${ }^{(51)}$.

The preventive effects of prebiotic feeding during perinatal and post-weaning periods in mouse models of allergies were analysed by studying biomarkers related to tolerance (IgG2a, IgA, interferon- $\gamma$, transforming growth factor- $\beta$ and IL-10), to allergies (IgE, IgG1, IL-4, IL-17 and symptoms) and to microbiota (propionate and MyD88) ${ }^{(52)}$. Regardless of diet, sensitised mice exhibited similar levels of IgE, IgG1, CD-23, IL-4, IL-17 and symptoms ${ }^{(52)}$. Prebiotic exposure during the perinatal and post-weaning periods induced the highest expression of biomarkers related to tolerance without affecting the biomarkers related to allergies ${ }^{(52)}$. A Cochrane review concluded that there is some evidence that a prebiotic supplement added to infant formula may prevent eczema ${ }^{(53)}$. A reduced occurrence of early atopic dermatitis in infants that had a low risk for atopy has also been reported ${ }^{(54)}$. In another recent study in 365 infants, no differences were observed in the incidence of allergies or infections ${ }^{(13)}$. In fact, a systematic review has concluded that the data available in relation to the use of prebiotics in infant formulas for the prevention of infections remain inconclusive ${ }^{(37)}$.

It is unclear whether the use of prebiotics should be restricted to infants who are at a high risk for allergies and whether they may also have an effect on other allergic diseases, including asthma ${ }^{(37)}$. Further research is needed before the routine use of prebiotics can be recommended for the prevention of allergies in formula-fed infants ${ }^{(37)}$. Data on symbiotic combinations are still limited.

\section{Conclusion}

Even if there is not enough evidence to state that supplementation of infant formula with prebiotics does result in a relevant clinical benefit ${ }^{(37)}$, there is evidence that prebiotics added to infant formula have beneficial effects on gut microbiota, metabolic activity, stool consistency and frequency and the development of some immune markers. Because prebiotics are present in breast milk as human milk OS, and because their addition to infant formula is safe, there seems to be no reason not to add them to infant formula, even though the evidence for the clinical benefit of doing so is limited. Future research should focus on specificity, safety, dosage and combinations. 


\section{References}

1. Kunz C, Rudloff S, Baier W, et al. (2000) Oligosaccharides in human milk: structural, functional, and metabolic aspects. Annu Rev Nutr 20, 699-722.

2. Harmsen HJ, Wildeboer-Veloo AC, Raangs GC, et al. (2000) Analysis of intestinal flora development in breast-fed and formula-fed infants by using molecular identification and detection methods. J Pediatr Gastroenterol Nutr 30, 61-71.

3. Johnson CL \& Versalovic J (2012) The human microbiome and its potential importance to pediatrics. Pediatrics 129, 950-960.

4. Gibson GR \& Roberfroid MB (1995) Dietary modulation of the human colonic microbiota: introducing the concept of prebiotics. J Nutr 125, 1401-1412.

5. Roberfroid M, Gibson GR, Hoyles L, et al. (2010) Prebiotic effects: metabolic and health benefits. Br J Nutr 104, Suppl. 2, S1-S63.

6. Yaron S, Shachar D, Abramas L, et al. (2013) Effect of high $\beta$-palmitate content in infant formula on the intestinal microbiota of term infants. J Pediatr Gastroenterol Nutr $\mathbf{5 6}$, 376-381.

7. Stewart ML, Timm DA \& Slavin JL (2008) Fructooligosaccharides exhibit more rapid fermentation than long-chain inulin in an in vitro fermentation system. Nutr Res 28, 329-334.

8. González R, Klaassens ES, Malinen E, et al. (2008) Differential transcriptional response of Bifidobacterium longum to human milk, formula milk, and galactooligosaccharide. Appl Environ Microbiol 74, 4686-4694.

9. Watson D, O'Connell Motherway M, Schoterman $\mathrm{MH}$, et al. (2013) Selective carbohydrate utilization by lactobacilli and bifidobacteria. J Appl Microbiol 114, 1132-1146.

10. Veereman-Wauters G, Staelens S, Van de Broek H, et al. (2011) Physiological and bifidogenic effects of prebiotic supplements in infant formulae. J Pediatr Gastroenterol Nutr 52, 763-771.

11. Knol J, Scholtens P, Kafka C, et al. (2005) Colon microflora in infants fed formula with galacto- and fructo-oligosaccharides: more like breast-fed infants. J Pediatr Gastroenterol Nutr 40, 36-42.

12. Scholtens PA, Alliet P, Raes M, et al. (2008) Fecal secretory immunoglobulin A is increased in healthy infants who receive a formula with short-chain galacto-oligosaccharides and longchain fructo-oligosaccharides. J Nutr 138, 1141-1147.

13. Sierra C, Bernal MJ, Blasco J, et al. (2015) Prebiotic effect during the first year of life in healthy infants fed formula containing GOS as the only prebiotic: a multicentre, randomised, double-blind and placebo-controlled trial. Eur J Nutr 54, 89-99.

14. Ben XM, Li J, Feng ZT, et al. (2008) Low level of galactooligosaccharide in infant formula stimulates growth of intestinal bifidobacteria and lactobacilli. World J Gastroenterol 14, 6564-6568.

15. Ben XM, Zhou XY, Zhao WH, et al. (2004) Supplementation of milk formula with galacto-oligosaccharides improves intestinal micro-flora and fermentation in term infants. Chin Med J (Engl) 117, 927-931.

16. Rinne MM, Gueimonde M, Kalliomäki M, et al. (2005) Similar bifidogenic effects of prebiotic-supplemented partially hydrolyzed infant formula and breastfeeding on infant gut microbiota. FEMS Immunol Med Microbiol 43, 59-65.

17. Fedorak RN \& Madsen KI (2004) Probiotics and prebiotics in gastrointestinal disorders. Curr Opin Gastroenterol 20, $146-155$.
18. Costalos C, Kapiki A, Apostolou M, et al. (2008) The effect of a prebiotic supplemented formula on growth and stool microbiology of term infants. Early Hum Dev 84, 45-49.

19. Scalabrin DM, Mitmesser SH, Welling GW, et al. (2012) New prebiotic blend of polydextrose and galacto-oligosaccharides has a bifidogenic effect in young infants. $J$ Pediatr Gastroenterol Nutr 54, 343-352.

20. Kapiki A, Costalos C, Oikonomidou C, et al. (2007) The effect of a fructo-oligosaccharide supplemented formula on gut flora of preterm infants. Early Hum Dev 83, 335-339.

21. Nakamura N, Gaskins HR, Collier CT, et al. (2009) Molecular ecological analysis of fecal bacterial populations from term infants fed formula supplemented with selected blends of prebiotics. Appl Environ Microbiol 75, 1121-1128.

22. Walton GE, van den Heuvel EG, Kosters MH, et al. (2012) A randomised crossover study investigating the effects of galacto-oligosaccharides on the faecal microbiota in men and women over 50 years of age. BrJ Nutr 107, 1466-1475.

23. Ladirat SE, Schuren FH, Schoterman MH, et al. (2014) Impact of galacto-oligosaccharides on the gut microbiota composition and metabolic activity upon antibiotic treatment during in vitro fermentation. FEMS Microbiol Ecol 87, 41-51.

24. Bakker-Zierikzee AM, Alles MS, Knol J, et al. (2005) Effects of infant formula containing a mixture of galacto- and fructooligosaccharides or viable Bifidobacterium animalis on the intestinal microflora during the first 4 months of life. $\mathrm{Br} \mathrm{J}$ Nutr 94, 783-790.

25. Rodriguez-Colinas B, Kolida S, Baran M, et al. (2013) Analysis of fermentation selectivity of purified galacto-oligosaccharides by in vitro human faecal fermentation. Appl Microbiol Biotechnol 97, 5743-5752.

26. Schmelzle H, Wirth S, Skopnik H, et al. (2003) Randomized double-blind study of the nutritional efficacy and bifidogenicity of a new infant formula containing partially hydrolyzed protein, a high beta-palmitic acid level, and nondigestible oligosaccharides. J Pediatr Gastroenterol Nutr 36, 343-351.

27. Williams T, Choe Y, Price P, et al. (2014) Tolerance of formulas containing prebiotics in healthy, term infants. J Pediatr Gastroenterol Nutr 59, 653-658.

28. Mugambi MN, Musekiwa A, Lombard M, et al. (2012) Synbiotics, probiotics or prebiotics in infant formula for full term infants: a systematic review. Nutr J 11, 81 .

29. Rao S, Srinivasjois R \& Patole S (2009) Prebiotic supplementation in full-term neonates: a systematic review of randomized controlled trials. Arch Pediatr Adolesc Med 163, 755-764.

30. Piemontese P, Giannì ML, Braegger CP, et al. (2011) Tolerance and safety evaluation in a large cohort of healthy infants fed an innovative prebiotic formula: a randomized controlled trial. PLOS ONE 6, e28010.

31. dos Santos EF, Tsuboi KH, Araújo MR, et al. (2011) Dietary galactooligosaccharides increase calcium absorption in normal and gastrectomized rats. Rev Col Bras Cir 38, 186-191.

32. Alliet P, Scholtens P, Raes M, et al. (2007) Effect of prebiotic galacto-oligosaccharide, long-chain fructo-oligosaccharide infant formula on serum cholesterol and triacylglycerol levels. Nutrition 23, 719-723.

33. Bruzzese E, Volpicelli M, Squeglia V, et al. (2009) A formula containing galacto- and fructo-oligosaccharides prevents intestinal and extra-intestinal infections: an observational study. Clin Nutr 28, 156-161.

34. Arslanoglu S, Moro GE, Schmitt J, et al. (2008) Early dietary intervention with a mixture of prebiotic oligosaccharides reduces the incidence of allergic manifestations and 
infections during the first two years of life. J Nutr 138 1091-1095.

35. Arslanoglu S, Moro GE \& Boehm G (2007) Early supplementation of prebiotic oligosaccharides protects formula-fed infants against infections during the first 6 months of life J Nutr 137, 2420-2424.

36. van Stuijvenberg M, Eisses AM, Grüber C, et al. (2011) Do prebiotics reduce the number of fever episodes in healthy children in their first year of life: a randomised controlled trial. Br J Nutr 106, 1740-1748.

37. Braegger C, Chmielewska A, Decsi T, et al. (2011) Supplementation of infant formula with probiotics and/or prebiotics: a systematic review and comment by the ESPGHAN committee on nutrition. J Pediatr Gastroenterol Nutr 52, 238-250.

38. Fukushima Y, Kawata Y, Hara H, et al. (1998) Effect of a probiotic formula on intestinal immunoglobulin A production in healthy children. Int J Food Microbiol 42, 39-44.

39. Yasui H, Mike A \& Ohwaki M (1989) Immunogenicity of Bifidobacterium breve and change in antibody production in Peyer's patches after oral administration. J Dairy Sci 72, $30-35$.

40. Seifert S \& Watzl B (2007) Inulin and oligofructose: review of experimental data on immune modulation. J Nutr $\mathbf{1 3 7}$ Suppl. 11, 2563S-2567S.

41. Field CJ, Van Aerde JE, Robinson LE, et al. (2008) Effect of providing a formula supplemented with long-chain polyunsaturated fatty acids on immunity in full-term neonates. Br J Nutr 99, 91-99.

42. Raes M, Scholtens PA, Alliet P, et al. (2010) Exploration of basal immune parameters in healthy infants receiving an infant milk formula supplemented with prebiotics. Pediatr Allergy Immunol 21, e377-e385.

43. Gopalakrishnan A, Clinthorne JF, Rondini EA, et al. (2012) Supplementation with galacto-oligosaccharides increases the percentage of NK cells and reduces colitis severity in Smad3-deficient mice. J Nutr 142, 1336-1342.

44. van Hoffen E, Ruiter B, Faber J, et al. (2009) A specific mixture of short-chain galacto-oligosaccharides and long-chain fructo-oligosaccharides induces a beneficial immunoglobulin profile in infants at high risk for allergy. Allergy 64, 484-487.

45. Bakker-Zierikzee AM, Tol EA, Kroes H, et al. (2006) Faecal SIgA secretion in infants fed on pre- or probiotic infant formula. Pediatr Allergy Immunol 17, 134-140.

46. Schouten B, Van Esch BC, Kormelink TG, et al. (2011) Nondigestible oligosaccharides reduce immunoglobulin free light-chain concentrations in infants at risk for allergy. Pediatr Allergy Immunol 22, 537-542.

47. Tanabe S \& Hochi S (2010) Oral administration of a galactooligosaccharide preparation inhibits development of atopic dermatitis-like skin lesions in NC/Nga mice. Int J Mol Med 25, 331-336.

48. Moro G, Arslanoglu S, Stahl B, et al. (2006) A mixture of prebiotic oligosaccharides reduces the incidence of atopic dermatitis during the first six months of age. Arch Dis Child 91, 814-819.

49. Williams HC \& Grindlay DJ (2010) What's new in atopic eczema? An analysis of systematic reviews published in 2007 and 2008. Part 2. Disease prevention and treatment. Clin Exp Dermatol 35, 223-227.

50. Vos AP, van Esch B, M'Rabet L, et al. (2007) Dietary supplementation with specific oligosaccharide mixtures decreases parameters of allergic asthma in mice. Int Immunopharmacol 6, 1277-1286.

51. Vos AP, Haarman M, Buco A, et al. (2006) A specific prebiotic oligosaccharide mixture stimulates delayed-type hypersensitivity in a murine influenza vaccination model. Int Immunopharmacol 6, 1277-1286.

52. Gourbeyre P, Desbuards N, Grémy G, et al. (2013) Perinatal and postweaning exposure to galactooligosaccharides/inulin prebiotics induced biomarkers linked to tolerance mechanism in a mouse model of strong allergic sensitization. J Agric Food Chem 61, 6311-6320.

53. Osborn DA \& Sinn JK (2013) Prebiotics in infants for prevention of allergy. The Cochrane Database of Systematic Reviews 2013, issue 3, CD006474.

54. Grüber C, van Stuijvenberg M, Mosca F, et al. (2010) Reduced occurrence of early atopic dermatitis because of immunoactive prebiotics among low-atopy-risk infants. J Allergy Clin Immunol 126, 791-797. 